\title{
Optimization of Monochromated TEM for Ultimate Resolution Imaging and Ultrahigh Resolution Electron Energy Loss Spectroscopy
}

Sergei Lopatin ${ }^{1 *}$, Bin Cheng ${ }^{2}$, Wei-Ting Liu ${ }^{2,3}$, Meng-Lin Tsai ${ }^{3}, \mathrm{Jr}^{-} \mathrm{Hau} \mathrm{He}^{2}$, Andrey Chuvilin ${ }^{4,5}$

${ }^{1}$ Imaging and Characterization Core Lab, King Abdullah University of Science and Technology, Thuwal, Saudi Arabia

${ }^{2}$ Computer, Elec, Math Sciences \& Eng, King Abdullah University of Science and Technology, Thuwal, Saudi Arabia

${ }^{3}$ Department of Materials Science and Engineering, National Tsing Hua University, Hsinchu, Taiwan

${ }^{4}$ CIC nanoGUNE Consolider, San Sebastian, Spain

${ }^{5}$ IKERBASQUE, Basque Foundation for Science, Bilbao, Spain

* Corresponding author, E-mail address: sergei.lopatin@kaust.edu.sa (Sergei Lopatin)

\begin{abstract}
The performance of a monochromated transmission electron microscope with Wien type monochromator is optimized to achieve an extremely narrow energy spread of electron beam and an ultrahigh energy resolution with spectroscopy. The energy spread in the beam is improved by almost an order of magnitude as compared to specified values. The optimization involves both the monochromator and the electron energy loss detection system. We demonstrate boosted capability of optimized systems with respect to ultra-low loss EELS and sub-angstrom resolution imaging (in a combination with spherical aberration correction).
\end{abstract}

KEYWORDS: EELS, ultra-low energy loss, phonons, plasmons, bandgap, sub-angstrom resolution

\section{Highlights:}

- New approach to optimize the performance of monochromated TEMs is proposed

- The approach is based on adjustments of already available combinations of FEI's monochromator and Gatan Imaging Filter

- TEM electron beam with $40 \mathrm{meV}$ and $600 \mathrm{pA}$ current is achieved

- EELS resolution of $26 \mathrm{meV}$ with $40 \mathrm{pA}$ probe current on a general purpose TEM is demonstrated

- Advantages for HR-TEM and TEM-EELS studies are evaluated 


\section{Introduction}

The Electron Energy Loss Spectroscopy (EELS) applied in Transmission Electron Microcopy (TEM) is a powerful analytical technique for characterization of materials. This technique is especially useful when TEM system is equipped with a monochromator of electron source in order to minimize the energy speared inherent to elector emission process. Conventionally the energy resolution of TEM-EELS system is defined as the full width at half maximum (FWHM) of the zero energy loss peak (ZLP) measured by the spectrometer. Typically non-dedicated monochromated TEM systems have the energy resolution in the range of $150-200 \mathrm{meV}$. At the same time, there is a considerable number of scientific challenges for which the key characteristic features of EELS measurements require the energy resolution better than $50 \mathrm{meV}$. Among those challenges are the vibrational spectroscopy for inorganic and organic materials [1], including the detection of hydrogen, measurement of phonons distribution [2], both local plasmon spectroscopy and spatial plasmon mapping [3] of nano scale objects such as resonant antennas [4], spatially resolved detection of bandgap states [5], and study of Van der Waals materials exhibiting intriguing structural, electronic and photonic properties [6]. Besides improving EELS energy resolution a strongly monochromated electron beam is a prerequisite for the high resolution imaging in TEM.

Recently a significant progress has been achieved in TEM-EELS performance. This progress is mainly associated with specialized systems such as ultrahigh vacuum dedicated scanning TEM plus a monochromator [2], for which a regular energy resolution of about $10 \mathrm{meV}$ is demonstrated, or one of a kind specially designed double Wien monochromator [7] for which $20 \mathrm{meV}$ is claimed. At the same time, it has been predicted theoretically, that the original design of a single Wien type monochromator implemented in general purpose high-end microscopes could deliver a comparable energy resolution $(16 \mathrm{meV})$ subject to the availability of a high resolution energy detection system [8].

The first successful attempt to extend the capabilities of a Wien type monochromator is already demonstrated for EELS application [3,6]. Here we report a detailed description of the optimization routine providing the ultimate energy resolution with all generations of Titan TEM-EELS systems from FEI Co (currently Thermo Fisher, USA) based on a combination of a monochromated gun and Gatan imaging filter (GIF) such as 966, 965 and 866. The optimized monochromated setup is tested for HR-TEM imaging and EELS applications.

\section{Method}

In general the performance of the TEM-EELS system is mainly determined by 2 factors: i) the energy speared of electrons in the beam (electrons produced by the gun and shaped into the beam by the condenser lenses) and ii) the energy resolution of EELS detection system, i.e. GIF. Both of 
these factors might also be effected by the overall system instability (microscope high tension fluctuations, mechanical vibrations, stray magnetic fields, etc.), which influence can be significantly reduced by shortening the data acquisition time.

To minimize the energy spread of the electron beam we first address the operation of a monochromator. FEI's standard monochromator is a Wien filter with crossed electric and magnetic fields perpendicular to the beam. The monochromator is placed between the Field Emission Gun (FEG) and the accelerator. The combination of three components: the electron extractor, the gun lens electrode and the monochromator, forms an effective electrostatic gun lens of the microscope. The difference between potentials of each component defines the strength of the effective gun lens and the resultant electron beam current (details see in [8]). For standard operation the potential of the gun lens electrode $V_{\text {gunlens }}=0.8 \mathrm{kV}$ is set lower compared to the extractor $V_{\text {extractor }}=4 \mathrm{kV}$ and the monochromator $V_{\text {mono }}=3 \mathrm{kV}$ potentials.

In case the monochromator is exited, it stretches the electron beam in the orthogonal direction and creates a dispersed image of the electron source or a dispersion line, which is essentially a distribution of electrons with respect to their energy. This line is focused at the exit plane of the monochromator, which is conjugated to the plane of energy selection slit positioned below the accelerator. The slit transmits only a small portion of electrons with selected narrow energy spread. According to the design [8] the excitation or the strength of the monochromator (i.e. the length of the dispersion line) is defined as $\Omega=E_{m} L /\left(\sqrt{8} V_{\text {mono }}\right)$, where $E_{m}$ is the monochromator electric deflection field and $L$ is the monochromator length.

As follows from the $\Omega$ definition, for a fixed value of the deflection field $E$, lowering of the monochromator potential $V_{\text {mono }}$ can result in significant increase of the monochromator strength (dispersion). Additionally, lowering $V_{\text {mono }}$ increases the overall strength of the effective electrostatic gun lens (since it depends on the difference between $V_{\text {extractor }}$ and combined effect of $V_{\text {gunlens }}$ and $V_{\text {mono }}$ ) reducing the electron source magnification and its contribution to the width of the dispersion line in non-dispersion direction. It also moves the gun lens focusing action further away from the electron source, reducing the electrons acceptance angle (Fig.1) and thus the effect of spherical and chromatic aberrations of the gun lens and parasitic aberrations such as coma. Smaller acceptance angle also causes less electrons passing through the gun lens to the monochromator lowering their Coulomb interactions. All these factors together suggest an increase of the monochromator filtering action under the lowering of $V_{\text {mono }}$ value.

The idea of the monochromator potential lowering was first tested by simulations of the electron beam trajectories for the key parameters of the microscope such as the high tension (HT), the extractor potential, etc. The simulations were performed by the "Raymono program" included in a standard software package of the monochromated TEMs from FEI Co. Indeed, we have 
established that lowering of the monochromator potential from $3000 \mathrm{~V}$ down to $400 \mathrm{~V}$ while keeping other parameters nearly the same significantly increases the monochromator dispersion (compare Fig.1a and b). Then we confirmed experimentally, that for the same size of the energy selection slit the setup with $400 \mathrm{~V}$ monochromator potential allows to filter electrons with the energy spread factor of 5-6 narrower compared to the standard settings. Further lowering of the monochromator potential does not lead to an additional dispersion enhancement.

According to our simulations ("Raymono program") the reduction of the extraction voltage $V_{\text {extractor }}$ results in effects similar to lowering of the monochromator potential: the electrons acceptance angle (Fig.1) gets even smaller, minimizing the effect of spherical and chromatic aberrations of the gun lens and parasitic aberrations such as coma. Even smaller acceptance angle also reduces the amount of electron passing through the gun lens to the monochromator minimizing Boersch effect. Thus, when the positive effect of lowering of the monochromator potential $V_{\text {mono }}$ reaches the intrinsic (design) limit further improvement should in principle be achievable by lowering the $V_{\text {extractor }}$. However, this also significantly reduces the total beam current (deliverable to a specimen). Even more, to prevent the FEG tip damage (so called "ring collapse") decreased $V_{\text {extractor }}$ needs to be accompanied by a reduction of the tip heating current (lower temperature of the tip) which is related to the manufacturer controlled FEG settings, and as such is not considered here.

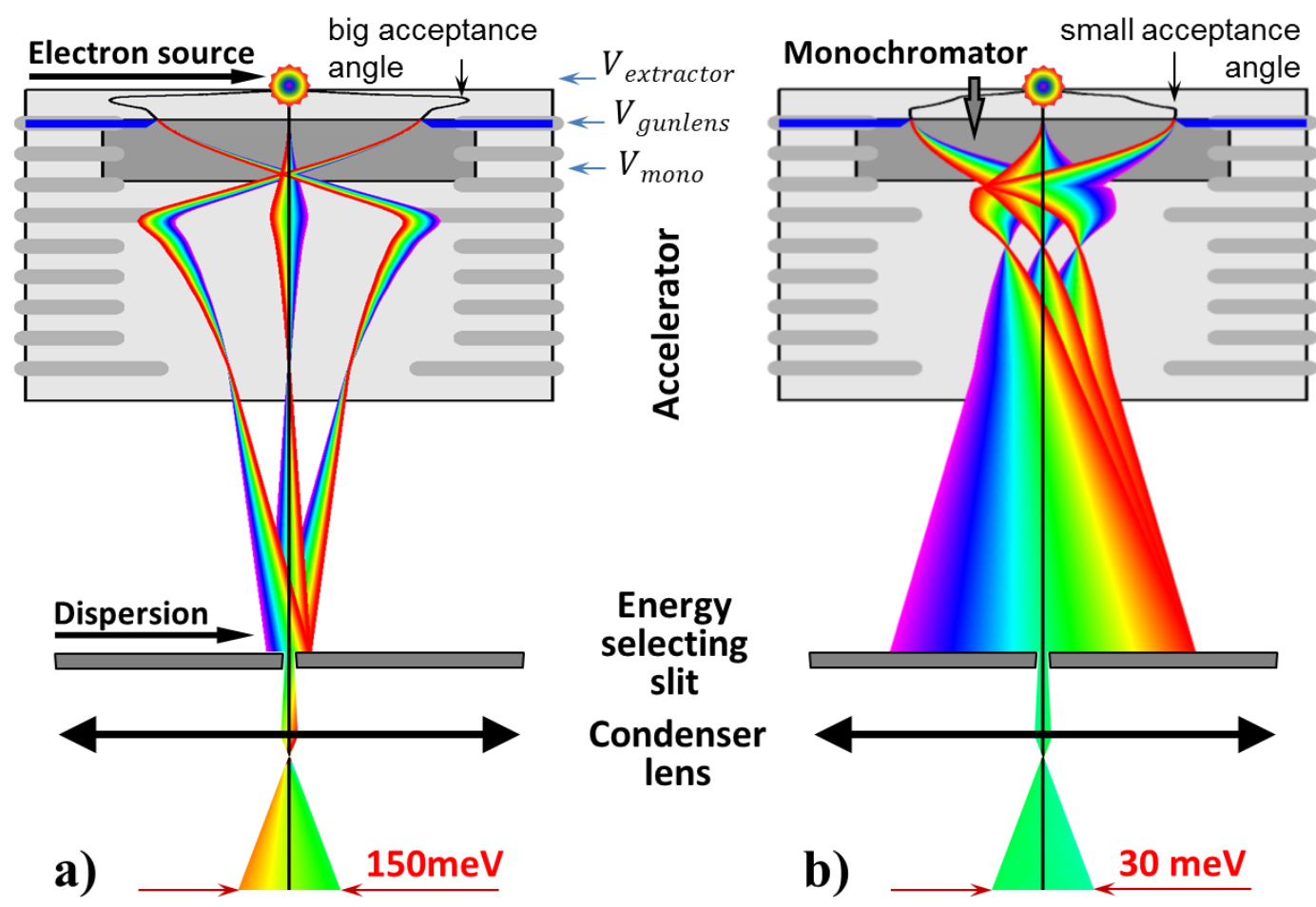

Figure 1. The simulation of the monochromated electron beam trajectories. Calculations are performed for $80 \mathrm{kV}$ of HT, monochromator excitation $1.4, V_{\text {extractor }}=3.85 \mathrm{kV}, V_{\text {gunlens }}=0.8 \mathrm{kV}$, the monochromator potential a) $3000 \mathrm{~V}$ and b) $400 \mathrm{~V}$. 
In order to measure the improved energy resolution we have also addressed the performance of the EELS detection system, namely the spectrum broadening due to the modulation transfer function (MTF) of the post-filter CCD camera. Usually such broadening is estimated as 3 times the energy width of a single channel. Thus, at the highest dispersion of $10 \mathrm{meV} / \mathrm{channel}$ typically available on most of the monochromated systems the resolution loss due to the CCD MTF is about $30 \mathrm{meV}$. We have implemented energy dispersions of 3 and $2 \mathrm{meV} / \mathrm{channel}$ (as measured by electrostatic drift tube of the GIF) to minimize the influence of MTF. The dispersion of $3 \mathrm{meV} / \mathrm{channel}$ allows observing relatively broad energy window in EELS which is suitable to study, for example, inter-band transitions or to measure precisely the bandgaps. The lower dispersion of $2 \mathrm{meV} / \mathrm{channel}$ is the most adequate for estimating the energy resolution of the beam and suitable for ultra-low electron energy loss spectroscopy in 0.1-2 eV range. Obtaining lower dispersions (2-3meV/channel) requires GIF post-slit lenses manipulations and normally can be performed by Gatan specialist upon a request.

The applicability of the modifications described above was verified on a number of Titan 60300 instruments both in TEM and STEM operation modes. In spite of substantially different configurations (low and high-base, 866, 965 and 966 GIFs) the energy resolution achieved was very close for all instruments. In STEM mode (microprobe with $1 \mathrm{mrad}$ semi-convergence angle) at $80 \mathrm{kV}$ HT the optimization routine described here allows to reach the energy resolution of $40 \mathrm{meV}$ with a probe current of $150 \mathrm{pA}$ on a regular basis (in the range from 0.1 to $5 \mathrm{~ms}$ exposure time). For a smaller probe current of $40 \mathrm{pA}(0.5 \mathrm{mrad}$ convergence angle) even a narrower energy spread of $30 \mathrm{meV}$ (and $26 \mathrm{meV}$ in a peak performance) was observed $(0.1 \mathrm{~ms}$ exposure time). Corresponding normalized ZLPs and time evolution of FWHM are shown in Fig. 2.

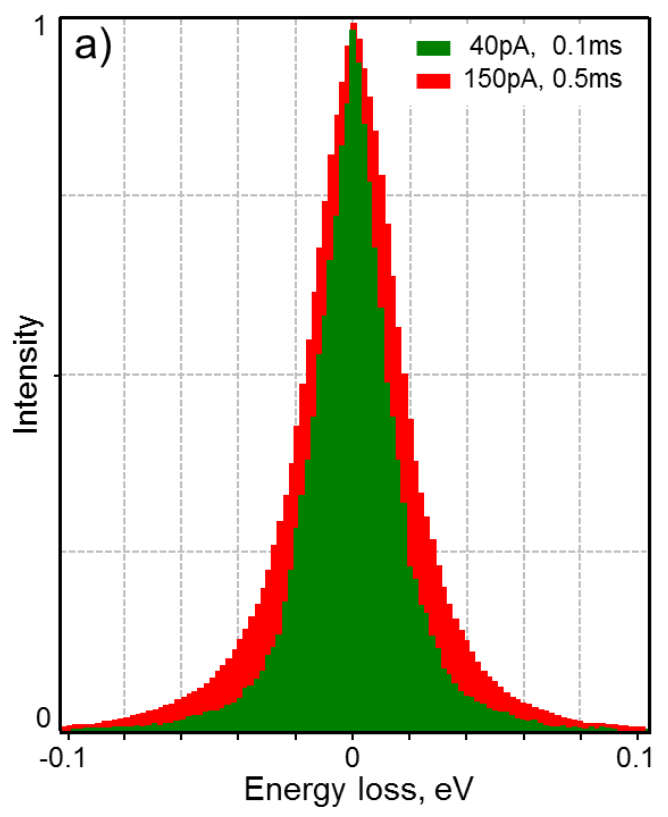

Energy loss, eV

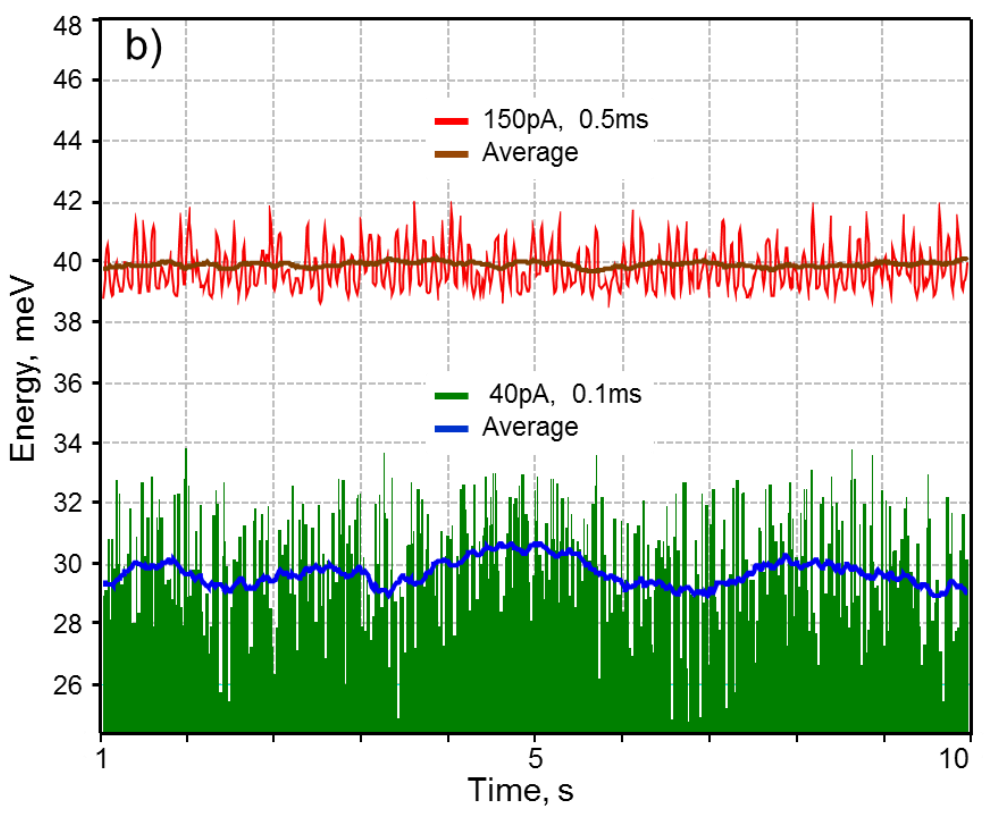

$-5-$ 
Figure 2. ZLP width measurements at $80 \mathrm{kV}$. a) Typical shape of ZLP for different probe current and exposition time. b) Tracking of the energy resolution (FWHM) with time.

In TEM mode at $80 \mathrm{kV}$ HT our measurements demonstrated that by following the monochromator optimization routine we can achieve the energy resolution of $40 \mathrm{meV}$ with unprecedented beam current of 600pA at $1 \mathrm{~ms}$ exposition time. At $100 \mathrm{~ms}$ exposition time the FWHM rises to $60 \mathrm{meV}$. Having in mind also the results obtained in STEM mode (ZLP width fluctuation, Fig.2b), the above achievements clearly point to the overall microscope instability as the main energy resolution limiting factor, and, on the other hand, showing the way to overcome it by increasing of temporal sampling of the acquisition.

Besides being useful for high resolution EELS analysis in case when spatial resolution is not required (big illumination area), such monochromated beam $(60 \mathrm{meV}$ in $0.1 \mathrm{sec}$ with $600 \mathrm{pA})$ at the same time allows to reduce significantly the effects of the chromatic aberration (especially at low voltages) in HR-TEM imaging and thus to achieve (in a combination with the correctors of spherical (Cs) aberration) sub-angstrom point-to-point resolution at low accelerating voltages.

\section{Application: TEM imaging}

The effect of the electron optics chromatic aberration on TEM resolution can be demonstrated by a comparison of damping envelopes for different energy spread of the beam at a given HT. Damping function is a cumulative measure of non-coherent aberrations of a TEM. Essentially it defines the upper frequency limit of the TEM contrast transfer function and thus defines the limit of information transfer through the optical system. In the systems with Cs corrector the corrected phase plate extends beyond the information limit and thus optimization of incoherent aberrations is the only way of improving point-to-point resolution. In this respect the effect of chromatic aberration, which contributes to overall damping envelope, can be minimized by decreasing the energy spread of the beam.
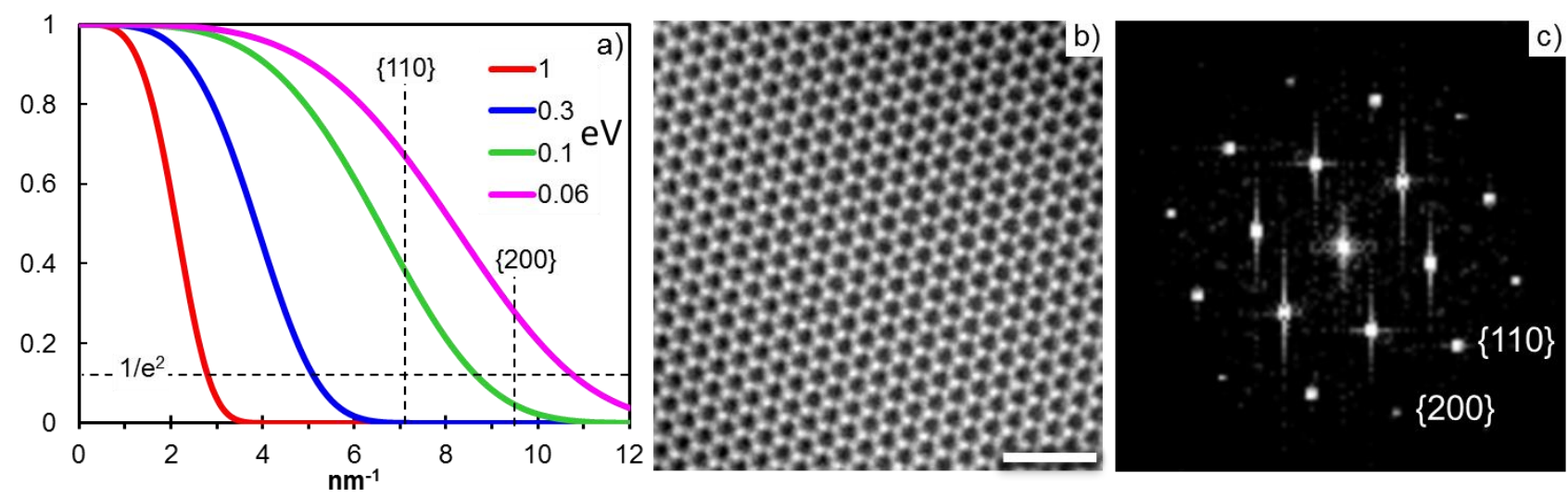
Figure 3. HR-TEM imaging of graphene at $80 \mathrm{kV}$ HT. a) Calculation of the damping envelope for $80 \mathrm{kV}$ TEM and various electron beam energy spreads from $1 \mathrm{eV}$ (red line) down to $60 \mathrm{meV}$ (purple). b) Unprocessed HR-TEM image of graphene obtained with optimized monochromated beam (scale bar is $1 \mathrm{~nm}$ ) at total exposition time of $2 \mathrm{~s}$, and c) corresponding FFT demonstrating spatial frequencies resolved.

As shown in Fig.3a for the energy spread of non-monochromated Schottky FEG (1eV) and cold FEG $(0.3 \mathrm{eV})$ the damping envelop for $80 \mathrm{keV}$ drops below the information limit threshold (usually taken as $1 / \mathrm{e}^{2}$ [9]) at spatial frequencies significantly lower than required, for example, for atomically resolved TEM imaging of graphene. Only $\{100\}$ lattice fringes of graphene can be observed with such a beam (see example in [10]). On the other hand, using the electron beam with the energy spread of about $100-200 \mathrm{meV}$ (monochromated) should in principal allow to resolve individual carbon atoms $(0.14 \mathrm{~nm},\{110\})$, see e.g. $[11,12]$. However, it is the transition to $60 \mathrm{meV}$ energy spread in the beam that enables the access to the spatial frequencies below $1 \AA$ and in case of graphene allows to resolve $0.105 \mathrm{~nm}\{200\}$ reliably as shown in Fig.3b,c. The most prominent consequence of such resolution increase is a proportional increase in contrast, which in its turn quadratically reduces the dose required to resolve single atoms, and that is of vital importance in a growing field of research in atomic dynamics [13].

HR-TEM imaging was performed with Gatan K2-IS camera on a high-base Titan 60-300 equipped with a high brightness electron gun (x-FEG), a monochromator, a Cs image corrector and Gatan 866 imaging filter. Round shape $1 \mu \mathrm{m}$ energy selecting aperture (standard FEI equipment for monochromated FEGs since 2010) was used. Such an aperture allows to have the microscope condenser system focused at the plane conjugated to the plane of the gun lens entrance aperture, providing the smallest beam size (highest intensity) and all the energies $(60 \mathrm{meV})$ mixed in the field of view. The image of graphene in Fig. $3 \mathrm{~b}$ was recorded as a series of 20 frames with $0.1 \mathrm{sec} / \mathrm{frame}$ exposure time and post-acquisition frame alignment.

\section{Application: EELS mapping of mid-IR plasmons}

Mapping of spatial distribution of surface plasmon polaritons (SPPs) in metal nano-resonators in visible energy range has become a hot topic following the rise of high resolution EELS methodology [14]. Utilization of the high energy resolution setup of a Titan microscope allowed to push plasmonic research to mid-IR range ( 170meV) [3]. 

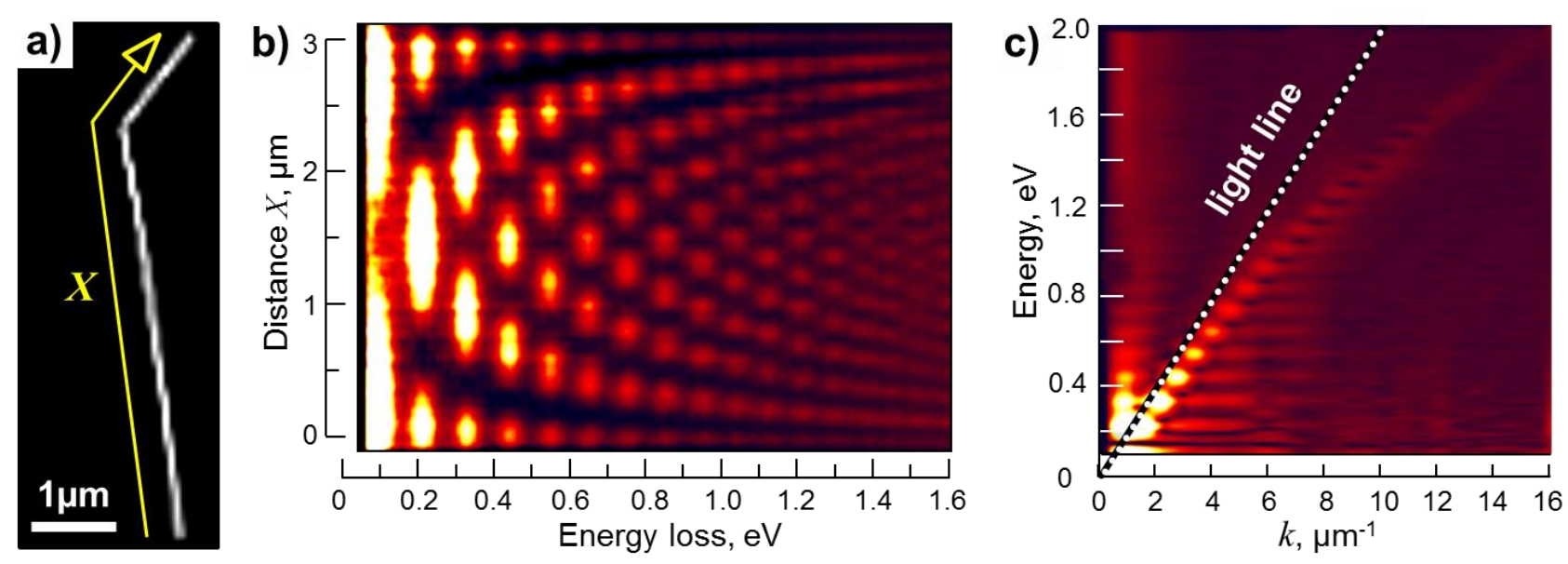

Figure 4. Silver nanowire surface plasmon mapping by a combination of STEM and HR EELS. a) Low magnification STEM image of the nanowire. Yellow arrow shows the direction of plasmon mapping. b) Spatial vs. energy distribution of SPP resonant maxima. c) The dispersion of resonant SPP waves in the nanowire obtained by 1D FFT of $b$ ). The intensity maximum curve corresponds to plasmon energy dependence on the wave number.

As shown in Fig. 4b, at optimized conditions plasmon mapping can be extended down to $\sim 100 \mathrm{meV}$ spectral range, which allows studying very fine structure of SPP resonant maxima distribution in energy space. Note that the $X$ direction of the map is "straitened" compared to the nanowire (Fig. 4a) since the distribution of resonant maxima was shown to be not effected by the nanowire bending [3]. Performing Fourier transformation on $X$ component of the plasmon map provides a direct visualization of resonant SPP dispersion as shown in Fig. 4c. Here the wave number defined as $k=\pi / X_{\max }$, where $X_{\max }$ is the distance between SPP maxima (since EELS maps intensity is square of amplitude). As expected, the obtained dispersion curve is positioned below the light line characterizing SPP as nonradiative, with the speed close to speed of light at low $k$.

Plasmon mapping in metal resonators is a relatively straightforward task due to high intensity of plasmon resonances. Orders of magnitude weaker phonon signals can also be detected and spatially mapped using proposed here optimization protocol [6]. Here we would like to emphasis, that besides similar performance can be achieved on a specialized low voltage HR EELS microscope [2], the instruments used in this study are the universal multipurpose tools able to realize HR TEM, HR STEM, tomography, holography, Lorentz mode, various diffraction techniques and anything else what is thinkable to be done in common modern TEM instrument in 60-300 keV beam energy range. 


\section{Application: EELS bandgap measurements}

Our approach for achieving the ultrahigh energy resolution EELS we applied for the investigation of electrical properties (bandgap variation) of layered two-dimensional $\mathrm{WSe}_{2}$ nanocrystals. Transition-metal dichalcogenide materials started to attract a significant interest due to their remarkable chemical and mechanical stability [15] and new physical properties through various quantum confinement effects [16]. For our study the sample of freestanding single/multiple layers $\mathrm{WSe}_{2}$ was produced by chemical vapor deposition growth and transferred to a holey carbon TEM grid subsequently.

The usage of EEL spectrum to determine the absolute value of the bandgap energy depends on how it is defined and which processing method is applied [17]. However, just to determine the difference in the bandgap between similar materials the majorities of methods should provide a reliable result as long as they consistently applied throughout all materials. We exploited a commonly used linear fit method for which the bandgap energy is estimated from the intersection of a linear fit to the conduction band onset on the loss spectrum with a zero level after the background subtraction. The later was performed by fitting of ZLP measured in vacuum. High signal-to-noise ratio was achieved by integration (after ZLP alignment) of $128 \mathrm{~K}$ spectra acquired at $3 \mathrm{meV}$ per channel dispersion with $0.5 \mathrm{~ms}$ per spectrum exposure time (cumulative acquisition time amounting to about $1 \mathrm{~min})$. The spectra were acquired in microprobe STEM mode with $0.5 \mathrm{mrad}$ semi convergence angle (about 9nm probe size), and 100pA probe current. To minimize the beam damage the probe was relocated (scanned) for every successive spectrum acquisition. The measurements were performed at $80 \mathrm{kV}$ with a high-base Titan 60-300 TEM equipped with a high brightness electron gun (x-FEG), a monochromator, a Cs probe corrector and Gatan Quantum 966 imaging filter.
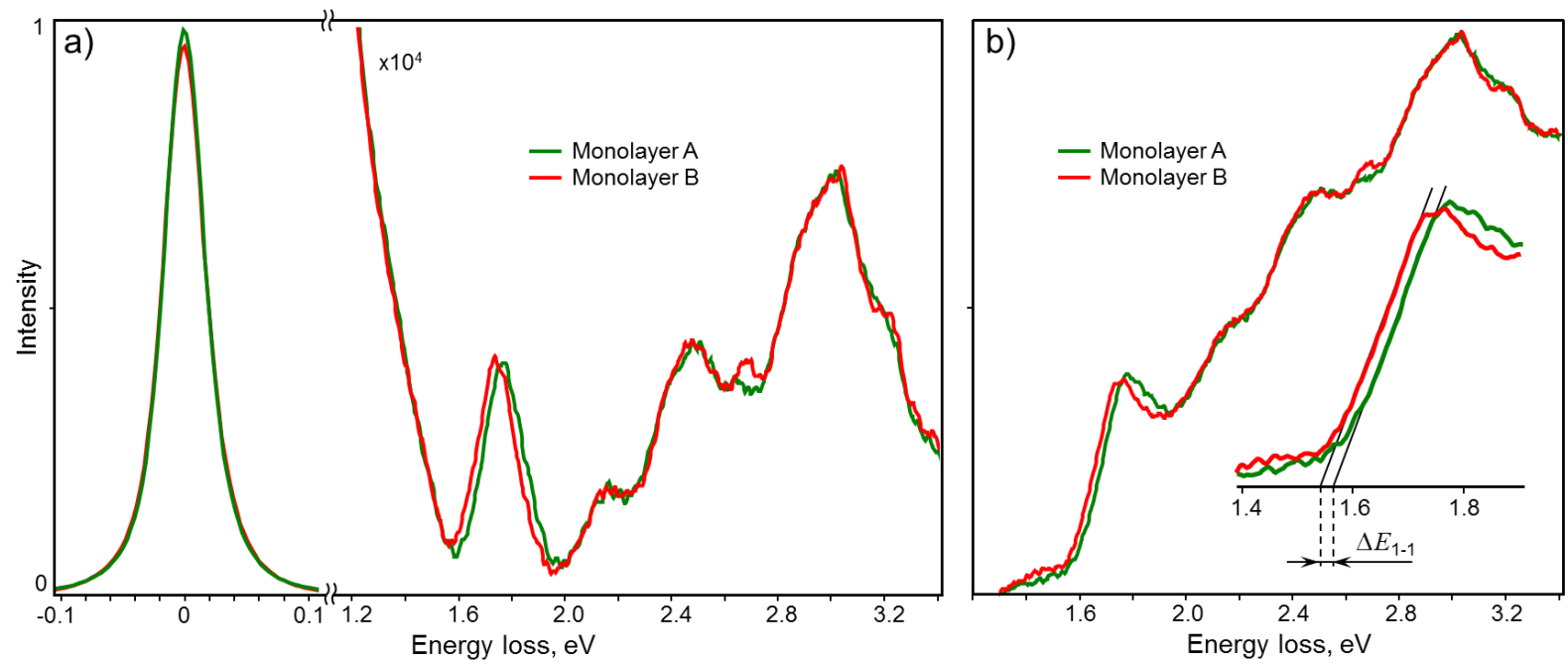

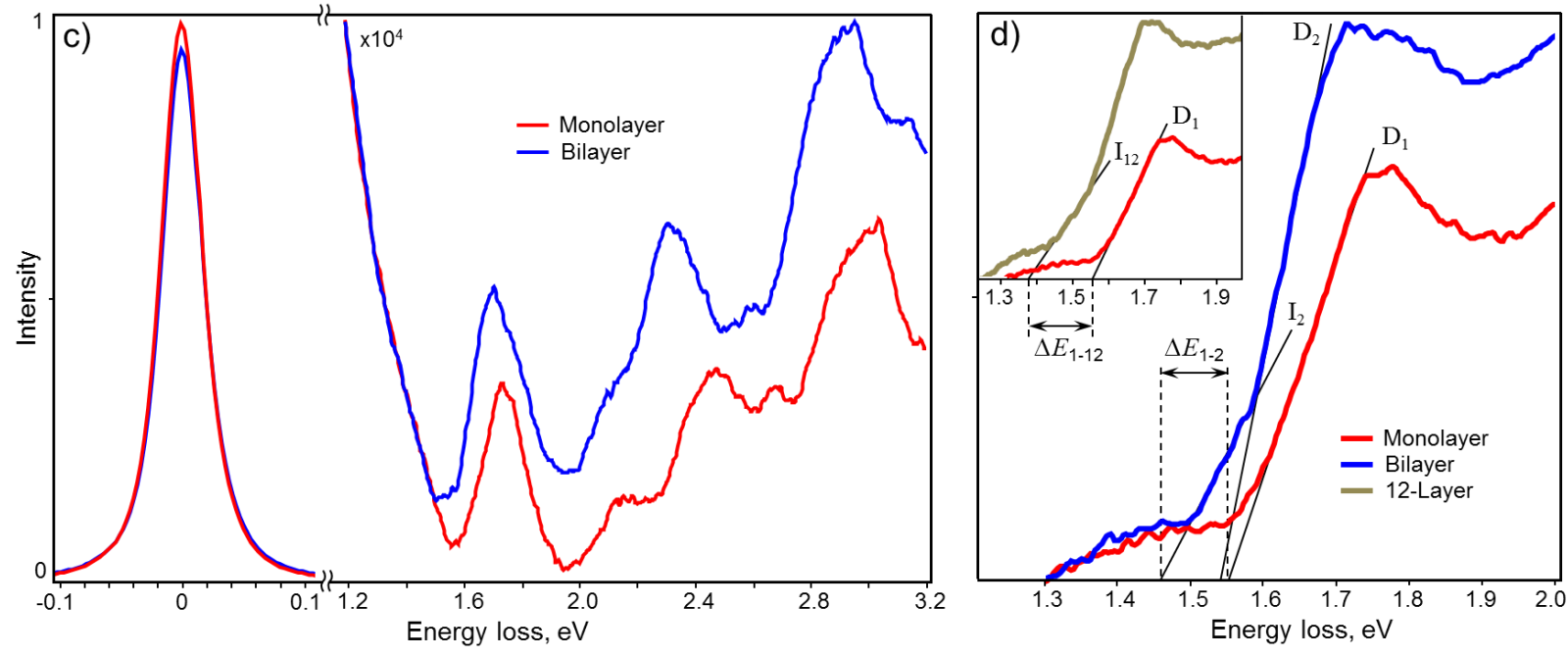

Figure 5. Ultra-high resolution EELS measurement of WSe 2 bandgap. a) and c) Integrated spectra from mono and bilayers. b) and d) Low loss signals after background subtraction to evaluate the bandgap difference.

First we consider a monolayer $\mathrm{WSe}_{2}$ known to be a direct gap semiconductor [18]. The integrated spectra obtained at different non-overlapping sample areas (A and B) are shown in Fig.5a. The conduction band onset (Fig.5b) demonstrates a linear increase of the intensity vs. the energy loss $E$ which is in remarkable contrast to the square root dependence $\left(\sim E^{0.5}\right)$ normally expected for a bulk direct gap semiconductor $[19,20]$. Furthermore, we observe a certain variation of the bandgap value measured at different areas of the monolayer $\mathrm{WSe}_{2}$. Owing to the ultrahigh energy resolution achievable with our approach even a very small bandgap difference can be detected. As shown both in Fig.5a and b, the two spectra from area A and B demonstrate nearly identical structure in the low loss region, except for the conduction band onset and corresponding local maximum. The inset in Fig.5b shows the linear fit intersections with the zero level to determine the bandgap difference $\Delta E_{1-1}$ which is found to be just $20 \pm 3 \mathrm{meV}$. This difference can be related to the variation of foreign atoms (mostly carbon) contamination on the surface and to the uniaxial strain [21] induced on a monolayer due to the sample preparation procedure [22].

Transition from a monolayer to a bilayer $\mathrm{WSe}_{2}$ reveals a red shift of the low loss spectral features as shown in Fig.5c. Moreover, the linear increase of the conduction band onset intensity with a single slope $\mathrm{D}_{1}$ specific for a monolayer is transformed into the linear increase (still) but with 2 distinct slopes $\mathrm{D}_{2}$ and $\mathrm{I}_{2}$ (Fig.5d). The bilayer slope $\mathrm{D}_{2}$ is apparently of the same physical origin as the monolayer slope $\mathrm{D}_{1}$. They both result in almost the same intersection with zero level and can be associated with a direct band gap electron excitation. On the other hand, the presence of the second slope $\left(\mathrm{I}_{2}\right)$ is indicative of the indirect gap transitions (by analogues with photoluminescence and 
photoconductivity measurements [13]) and direct-to-indirect band-gap crossover from a monolayer to bilayer $\mathrm{WSe}_{2}$. The bandgap in this case can be measured by the intersection of the linear fit to the slope $\mathrm{I}_{2}$ with the zero level (Fig.5d) which results in the mono-to-bilayer bandgap difference of $\Delta E_{1-2}=90 \pm 3 \mathrm{meV}$. Further increase of the number of layers qualitatively demonstrates the same split into direct and indirect slopes of the conduction bandgap onset accompanied by an additional red-shift of spectral features. For example, for 12-layers $\mathrm{WSe}_{2}$ (see the insert in Fig.5d) the bandgap difference with the monolayer is $\Delta E_{1-12}=183 \pm 3 \mathrm{meV}$.

It should be noted that in contrary to photoluminescence method of the bandgap determination for which the overall signal coming from a few-layer material is orders of magnitude weaker than the intensity from bilayer and monolayer [19], the EELS signal from a monolayer is the weakest.

\section{Conclusions}

In this study we demonstrate that the performance of existing common use monochromated TEM-EELS systems can be significantly improved approaching those of specialized instruments. The key factor for such an improvement is the deviation from a standard monochromator operation mode towards optimized settings demonstrated here experimentally. The electron beam with an enhanced monochromaticity in a combination with $\mathrm{Cs}$ image corrector allows to access subangstrom spatial frequencies in HR-TEM imaging even at low accelerating voltages without chromatic aberration correction. In a combination with high-resolution energy loss detection system the enhanced monochromated TEM system can be used to reliably differentiate EEL spectral features with just $20 \mathrm{meV}$ separation (down to $7 \mathrm{meV}$ as in [6]).

The optimization method reported here is straightforwardly applicable to the majority of monochromated FEI's Titan transmission electron microscopes. The number of such microscopes currently installed at different research facilities is considerably higher than one hundred. As our study clearly demonstrates the capabilities of these instruments in terms of both energy and spatial resolution can be significantly extended without hardware modifications. We do believe that herein reported results on the energy resolution optimization of TEM-EELS systems are useful and interesting for the scientific community.

\section{Acknowledgments}

Authors would like to thank Dr. Peter C Tiemeijer from FEI Co for fruitful comments and discussions regarding the monochromator operation and optimization. Also we would like to acknowledge Dmitry Melnikau for providing a sample of Ag nanowires. 


\section{References}

[1] Peter Rez, Toshihiro Aoki, Katia March, Dvir Gur, Ondrej L. Krivanek, Niklas Dellby, Tracy C. Lovejoy, Sharon G. Wolf \& Hagai Cohen, Damage-free vibrational spectroscopy of biological materials in the electron microscope, Nature Communications 7, 10945 (2016) doi:10.1038/ncomms10945

[2] Ondrej L. Krivanek, Tracy C. Lovejoy, Niklas Dellby, Toshihiro Aoki, R. W. Carpenter, Peter Rez, Emmanuel Soignard, Jiangtao Zhu, Philip E. Batson, Maureen J. Lagos, Ray F. Egerton, Peter A. Crozier, Vibrational spectroscopy in the electron microscope, Nature 514, (2014), pp. 209-212 doi:10.1038/nature13870

[3] David Rossouw and Gianluigi A. Botton, Plasmonic Response of Bent Silver Nanowires for Nanophotonic Subwavelength Waveguiding, Phys. Rev. Lett. 110, 066801 (2013) doi: https://doi.org/10.1103/PhysRevLett.110.066801

[4] Florian Huth, Andrey Chuvilin, Martin Schnell, Iban Amenabar, Roman Krutokhvostov, Sergei Lopatin, Rainer Hillenbrand, Resonant antenna probes for tip-enhanced infrared near-field microscopy, Nano Lett. 13, (2013), pp. 1065-1072 doi: $10.1021 / \mathrm{nl} 304289 \mathrm{~g}$

[5] W.J. Bowmana, K. Marchb, C.A. Hernandeza, P.A. Crozier, Measuring bandgap states in individual non-stoichiometric oxide nanoparticles using monochromated STEM EELS: The Praseodymium-ceria case, Ultramicroscopy 167, (2016), pp. 5-10 http://dx.doi.org/10.1016/j.ultramic.2016.04.009

[6] Alexander A. Govyadinov, Andrea Konecna, Andrey Chuvilin, Saül Vélez, Irene Dolado, Alexey Nikitin, Sergei Lopatin, Fèlix Casanova, Luis E. Hueso, Javier Aizpurua, Rainer Hillenbrand, Probing low-energy hyperbolic polaritons in van der Waals crystals with an electron microscope, Nature communication, 2017; 8: 95 doi: 10.1038/s41467-017-00056-y

[7] Luiz H. G. Tizei, Yung-Chang Lin, Masaki Mukai, Hidetaka Sawada, Ang-Yu Lu, Lain-Jong Li, Koji Kimoto, and Kazu Suenaga, Exciton Mapping at Subwavelength Scales in Two-Dimensional Materials, Phys. Rev. Lett. 114, 107601 (2015) doi: https://doi.org/10.1103/PhysRevLett.114.107601 
[8] P C Tiemeijer, Operation modes of a TEM monochromator, Inst. Phys. Conf. Ser. Vol. 161. (1999), pp.191-194

[9] M. A. O’Keefe, "Resolution” in high-resolution electron microscopy, Ultramicroscopy 47, (1992), pp. 282-297 https://doi.org/10.1016/0304-3991(92)90203-V

[10] Cristina Gómez-Navarro, Jannik C Meyer, Ravi S Sundaram, Andrey Chuvilin, Simon Kurasch, Marko Burghard, Klaus Kern, Ute Kaiser, Atomic structure of reduced graphene oxide, Nano Lett., 10 (4), (2010), pp. 1144-1148

doi: 10.1021/n19031617

[11] Jannik C. Meyer, C. Kisielowski, R. Erni, Marta D. Rossell, M. F. Crommie and A. Zettl, Direct Imaging of Lattice Atoms and Topological Defects in Graphene Membranes, Nano Lett., 8, (2008), pp. 3582-3586

doi: $10.1021 / \mathrm{nl} 801386 \mathrm{~m}$

[12] Çağlar Ö. Girit, Jannik C. Meyer, Rolf Erni, Marta D. Rossell, C. Kisielowski, Li Yang, CheolHwan Park, M. F. Crommie, Marvin L. Cohen, Steven G. Louie, A. Zettl, Graphene at the Edge: Stability and Dynamics, Science Vol. 323, 5922 (2009), pp. 1705-1708 doi: 10.1126/science.1166999

[13] Stephen T Skowron, Victor O Koroteev, Matteo Baldoni, Sergei Lopatin, Amaia Zurutuza, Andrey Chuvilin, Elena Besley, Reaction kinetics of bond rotations in graphene, Carbon, 105, (2016), pp.176-182 http://dx.doi.org/10.1016/j.carbon.2016.04.020

[14] F. Hofer, F. P. Schmidt, W. Grogger, G. Kothleitner, Fundamentals of electron energy-loss spectroscopy, IOP Conf. Series: Materials Science and Engineering 109 (2016), 012007 doi: 10.1088/1757-899X/109/1/012007

[15] Coleman JN, Lotya M, O'Neill A, Bergin SD, King PJ, Khan U, Young K, Gaucher A, De S, Smith RJ, Shvets IV, Arora SK, Stanton G, Kim HY, Lee K, Kim GT, Duesberg GS, Hallam T, Boland JJ, Wang JJ, Donegan JF, Grunlan JC, Moriarty G, Shmeliov A, Nicholls RJ, Perkins JM, 
Grieveson EM, Theuwissen K, McComb DW, Nellist PD, Nicolosi V., Two-dimensional nanosheets produced by liquid exfoliation of layered materials, Science 331 (2011), pp.568-571 doi: 10.1126/science. 1194975

[16] Kin Fai Mak, Changgu Lee, James Hone, Jie Shan, Tony F. Heinz, Atomically Thin MoS 2 : A New Direct-Gap Semiconductor, Phys. Rev. Lett. 105, 136805 (2010)

doi:https://doi.org/10.1103/PhysRevLett.105.136805

[17] S. Schamm, G. Zanchi, Study of the dielectric properties near the band gap by VEELS: gap measurement in bulk materials, Ultramicroscopy 96 (2003), pp.559-564 doi: 10.1016/S0304-3991(03)00116-5

[18] A. Kumar, P.K. Ahluwalia, Electronic structure of transition metal dichalcogenides monolayers $1 \mathrm{H}-\mathrm{MX}_{2}(\mathrm{M}=\mathrm{Mo}, \mathrm{W} ; \mathrm{X}=\mathrm{S}, \mathrm{Se}, \mathrm{Te})$ from ab-initio theory: new direct band gap semiconductors, Eur. Phys. J. B 85, 186 (2012) doi: 10.1140/epjb/e2012-30070-x

[19] B. Rafferty, L. M. Brown, Direct and indirect transitions in the region of the band gap using electron-energy-loss spectroscopy, Phys. Rev. B 58, 10326 (1998) doi: https://doi.org/10.1103/PhysRevB.58.10326

[20] S. Lazar, G.A. Botton, M.-Y. Wu, F.D. Tichelaar, H.W. Zandbergen, Materials science applications of HREELS in near edge structure analysis and low-energy loss spectroscopy, Ultramicroscopy 96 (2003), pp.535-546 doi: 10.1016/S0304-3991(03)00114-1

[21] Chung-Huai Chang, Xiaofeng Fan, Shi-Hsin Lin, and Jer-Lai Kuo, Orbital analysis of electronic structure and phonon dispersion in $\mathrm{MoS}_{2}, \mathrm{MoSe}_{2}, \mathrm{WS}_{2}$, and $\mathrm{WSe}_{2}$ monolayers under strain, Phys. Rev. B 88, 195420 (2013)

doi: https://doi.org/10.1103/PhysRevB.88.195420

[22] H. Sahin, S. Tongay, S. Horzum, W. Fan, J. Zhou, J. Li, J. Wu, F. M. Peeters, Anomalous Raman spectra and thickness-dependent electronic properties of $\mathrm{WSe}_{2}$, Phys. Rev. B 87, 165409 (2013) 
doi: https://doi.org/10.1103/PhysRevB.87.165409 